\section{Enlargement of the Inferior Intercavernous Sinus: A New Sign for the Diagnosis of Craniospinal Hypotension}

We read with interest the recent article entitled "MR Imaging of the Optic Nerve Sheath in Patients with Craniospinal Hypotension" by Rohr et al. ${ }^{1}$ In patients with craniospinal hypotension, apart from engorged venous sinuses, enlarged pituitary gland, subdural effusions, and sagging of the brain, the authors have observed a partially or fully collapsed intersheath space of the optic nerves. Such a demonstration is particularly clear in their Fig 3 . We would like to draw more attention to changes in the pituitary gland. As shown in Fig $3 C$ (arrow), enlargement of the sellar content is also due partially to dilation of the inferior intercavernous sinus (IICS), which has collapsed after treatment. Such a dilation is not mentioned in the legend. In our experience, the IICS, which is visible in roughly $30 \%$ of children on MR imaging, is never quite visible in the healthy adult.

In a personal series of 12 adult patients with intracranial hypotension syndrome studied by MR imaging, the IICS was visualized in 11 cases $^{2}$ : The IICS was very thin in 2 cases, prominent in 6 cases, and very thick $(>3 \mathrm{~mm}$ ) in 3 cases (Fig 1 ). The differential diagnosis includes a pituitary gland with increased height on one hand, corresponding to physiologic (hyperplasia, pregnancy, small sella) or pathologic conditions (a holosellar pituitary adenoma, hypophysitis, and so forth), and focal intrasellar lesions such as pituitary microadenoma on the other hand. Recognition of the presence of the IICS allows exclusion of these pituitary abnormalities. In 3 of 12 cases of our series, MR imaging follow-up after blood patch or spontaneous resolution of intracranial hypotension demonstrated a significant decrease in the height of the IICS (Fig 2). In our experience, dilation of the IICS constitutes a useful adjunctive sign for the diagnosis of intracranial hypotension. Moreover, enlargement of the IICS should be added to the other causes of enlargement of the sellar content.

\section{References}

1. Rohr A, Jensen U, Riedel C, et al. MR imaging of the optic nerve sheath in patients with craniospinal hypotension. AJNR Am $J$ Neuroradiol 2010;31:1752-57

2. Cattin F, Billon-Grand NC, David A, et al. Enlargement of the inferior inter-

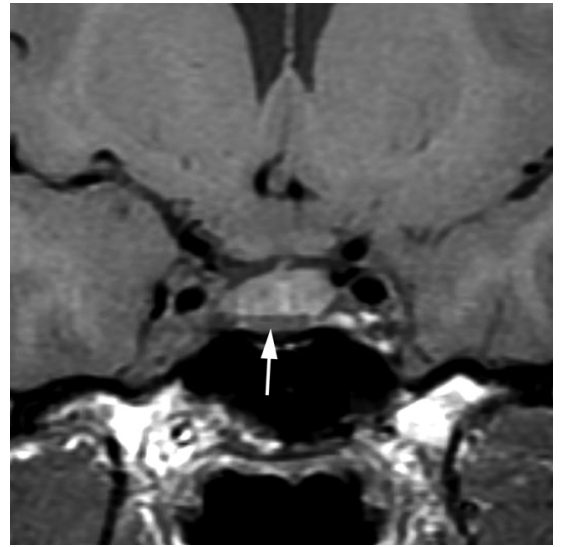

Fig 1. A 21-year-old woman with orthostatic headaches after lumbar puncture. Prominent IICS doubling of the sellar floor (arrow) and bulging of the pituitary gland.

cavernous sinus: a new sign of intracranial hypotension syndrome. In: Proceedings of the American Society of Neuroradiology 48th Annual Meeting and Neuroradiology Education and Research Foundation Symposium, Boston, Massachusetts May 15-20, 2010

J.-F. Bonneville

F. Cattin

Jean Minjoz Hospital

Department of Neuroradiology

Besançon, France

F. Bonneville

Rangueil Hospital

Department of Neuroradiology

Toulouse, France

http://dx.doi.org/10.3174/ajnr.A2816
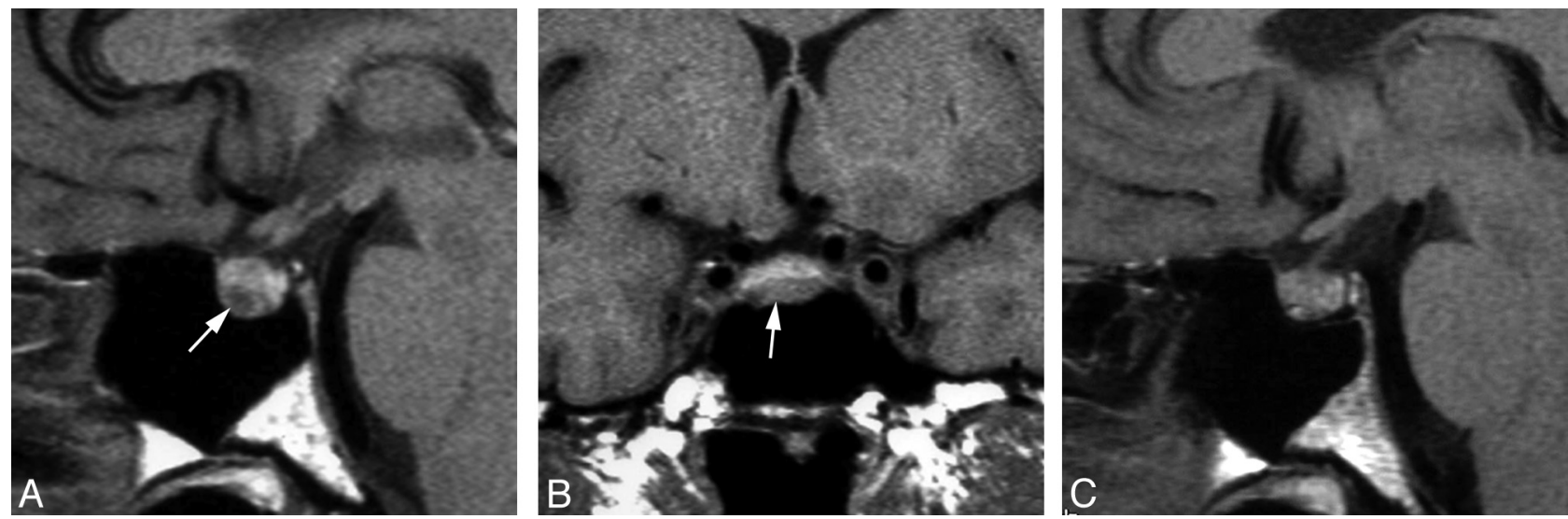

Fig 2. A 46-year-old man with acute Iymphoblastic leukemia. Iterative lumbar punctures. An enlarged IICS, simulating a hypointense focal intrasellar lesion on $T 1$ images (A and B) (arrow) which has collapsed after treatment $(C)$. 